UCTN

Endoscopic mucosal resection techniques have now evolved to include endoscopic submucosal dissection [1-3]. Although this technique enables larger tumors to be resected en bloc with adequate margins, a high complication rate due to bleeding, especially arterial bleeding, has become apparent [4]. In a considerable number of cases of bleeding that occur during endoscopic submucosal dissection the bleeding source cannot be identified because of a blood-filled field. The hemostatic method used during endoscopic submucosal dissection should secure a sufficient visual field to see the bleeding lesion, should be easy to perform, and should be capable of reliably stopping arterial bleeding. With these requirements in mind, we have developed a method of endoscopic hemostasis for use during endoscopic submucosal dissection that consists of high-frequency hemostatic forceps (HDB2422W; Pentax, Tokyo, Japan) (Figure $\mathbf{1} \mathbf{a}$ ) and an endoscope equipped with a water-jet system (Pentax EG2931/SA-P2) (Figure 1 b).

The forceps have been developed specially for hemostasis and have a narrow opening angle, a small cup, and a blunt edge, to ensure efficient electrocoagulation. Coagulation of the bleeding vessel requires only a low electrical current, which rarely causes perforation. After pinpoint holding and mechanical compression, electrocoagulation is easily performed. The water-jet system supplies a continuous jet of water at high pressure, which easily and swiftly washes away any blood that is obstructing the visual field, allowing identification of the bleeding source. Because the water-jet system supplies water through an exclusive port, the forceps channel can be used for the hemostatic instrument. By washing the bleeding field with the water jet, the bleeding source can be immediately identified and coagulated (Figure 2, Video 1), although in a small number of cases of erupting venous bleeding it can be difficult to identify the bleeding source. There are several hemostatic procedures which

\title{
Novel endoscopic hemostasis technique for use during endoscopic submucosal dissection
}

are used to control arterial bleeding during and after endoscopic submucosal dissection, but this procedure is quite effective and can be used as the method of first choice for treating this complication.

\section{Endoscopy_UCTN_Code_TTT_1AO_2AD}

\section{S. Enomoto ${ }^{1,2}$, N. Yahagi², M. Fujishiro' ${ }^{2}$, M. Oka ${ }^{2}$, N. Kakushima ${ }^{2}$, M. Iguchi', K. Yanaoka ${ }^{1}$, K. Arii', H. Tamai', Y. Shimizu', M. Omata ${ }^{2}$, M. Ichinose ${ }^{1}$ ${ }^{1}$ Second Department of Internal Medi- cine, Wakayama Medical University, Wakayama, Japan \\ 2 Department of Gastroenterology, Facul- ty of Medicine, University of Tokyo, To- kyo, Japan.}

\section{References}

${ }^{1}$ Yahagi N, Fujishiro M, Kakushima $\mathrm{N}$ et al. Endoscopic submucosal dissection for early gastric cancer using the tip of an electrosurgical snare (thin type). Dig Endosc 2004; 16: 34- 38

2 Ono H, Kondo H, Gotoda T et al. Endoscopic mucosal resection for treatment of early gastric cancer. Gut 2001; 48: 225-229

${ }^{3}$ Yamamoto H, Seike Y, Higashizawa T et al. Successful en bloc resection of a large superficial gastric cancer by using sodium hyaluronate and electrocautery incision forceps. Gastrointest Endosc 2001; 54: 629-632

${ }^{4}$ Ohkuwa M, Hosokawa K, Boku N et al. New endoscopic treatment for intramucosal gastric tumors using an insulated-tip diathermic knife. Endoscopy 2001; 33: 221 - 226

Video The combination of high-frequency hemostatic forceps and a water-jet system that provides a continuous supply of water at high pressure swiftly washes away obstructing blood in the visual field and allows accurate and effective hemostasis. This method is quite effective for controlling arterial bleeding during and after endoscopic submucosal dissection.

online content including video sequences viewable at:

www.thieme-connect.de/ejournals/

abstract/endoscopy/doi/

$10.1055 / s-2006-925254$

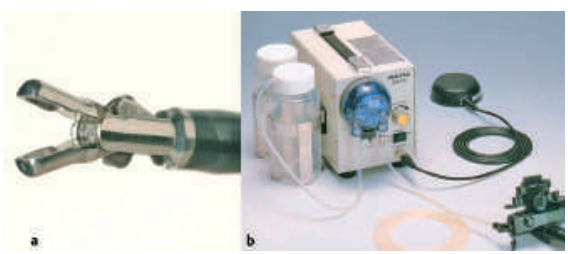

Figure 1 a The high-frequency hemostatic forceps (Pentax HDB2422W) has a narrow opening angle, small cup, and blunt edges. $\mathbf{b}$ The endoscope was fitted with a water-jet system (Pentax EG-2931/SA-P2): the water jet is delivered to the visual field by stepping on the foot switch.

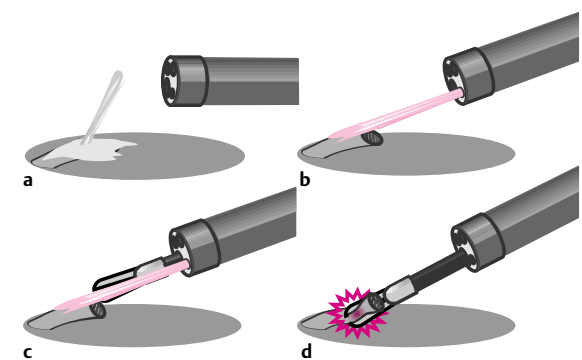

Figure 2 Schematic representation of the hemostatic procedure designed for use during endoscopic submucosal dissection, using hemostatic forceps and an endoscope equipped with a water-jet system. a Pulsatile bleeding from an exposed ulcer. b The water-jet system is used to clear the field in order to identify the source of bleeding and confirm its severity. c The hemostatic forceps are inserted via the forceps channel as water is delivered continuously at high pressure. $\mathbf{d}$ Electrocoagulation is performed after pinpoint holding and mechanical compression with the hemostatic forceps.

\section{Corresponding author}

\section{S. Enomoto, M.D.}

Second Department of Internal Medicine Wakayama Medical University, 811-1, Kimiidera, Wakayama 641-0012, Japan

Fax: $\quad$ +81-73-445-3616

Email: shoe@orion.ocn.ne.jp 